\title{
Interferenti endocrini e funzione gonadica: focus su steroidogenesi testicolare e infertilità maschile
}

\author{
Cristina de Angelis ${ }^{1} \cdot$ Marco Mazzella ${ }^{1} \cdot$ Rosario Pivonello ${ }^{1,2}$
}

Accettato: 10 novembre 2021 / Pubblicato online: 26 gennaio 2022

(c) The Author(s) 2022

\section{Sommario}

Evidenze sperimentali in modelli animali e in vitro dimostrano un ruolo deleterio degli interferenti endocrini (IE) sulla gonade maschile; tuttavia, nonostante vi siano alcune evidenze osservazionali di associazione tra alterazioni seminali e/o endocrine ed esposizione ad alcuni IE, in particolare quelli ad azione anti-androgenica, pro-estrogenica o capaci di indurre stress ossidativo, non è tuttora dimostrabile l'inferenza di casualità nell'uomo, e permane una vasta area di incertezza. La rassegna fornisce una panoramica sinottica delle evidenze cliniche relative alle alterazioni di spermatogenesi e steroidogenesi testicolare in relazione all'esposizione a selezionati IE, con particolare riferimento all'esposizione in età adulta.

Parole chiave Interferenti endocrini $\cdot$ Testicolo $\cdot$ Spermatogenesi $\cdot$ Testosterone

\section{Interferenti endocrini: definizione, classificazione e meccanismi d'azione}

L'infertilità è una condizione patologica a eziologia complessa, definita dall'Organizzazione Mondiale della Sanità come l'assenza di concepimento dopo almeno 12 mesi di regolari rapporti sessuali mirati non protetti; un fattore maschile di infertilità, isolato $\mathrm{o}$ in combinazione con fattore femminile, sottende alla condizione di infertilità di coppia in circa il 50\% dei casi [1]. La progressiva riduzione globale della fertilità di coppia e della qualità del liquido seminale documentata a partire dagli anni '30 [2] e la netta evidenza di un'eterogeneità geografica della qualità del liquido semi-

\author{
Proposto da D. Gianfrilli. \\ $凶$ R. Pivonello \\ rosario.pivonello@unina.it \\ C. de Angelis \\ cristinadeangelis83@ hotmail.it \\ M. Mazzella \\ marco.mazzella.mm@gmail.com \\ 1 Dipartimento di Medicina Clinica e Chirurgia, Sezione di \\ Endocrinologia, Unità di Andrologia e Medicina della \\ Riproduzione e della Sessualità Maschile e Femminile \\ (FERTISEXCARES), Università Federico II di Napoli, Napoli, \\ Italia \\ 2 Staff of Unesco Chair for Health Education and Sustainable \\ Development, Federico II University, Napoli, Italia
}

nale hanno suggerito che tali cambiamenti siano potenzialmente e parzialmente ascrivibili anche a fattori ambientali locali, compresi stile di vita ed esposizione ambientale/occupazionale a inquinanti, oltre che a fattori genetici [3]. Tra gli inquinanti ambientali, gli IE, definiti come sostanze chimiche o miscele di sostanze chimiche esogene che interferiscono con qualsiasi aspetto dell'azione ormonale, compresa sintesi, secrezione, trasporto, metabolismo, attività di legame a recettori/target ed eliminazione degli ormoni, inducendo effetti avversi in un organismo o nella sua progenie [4], sono stati oggetto negli ultimi due decenni di intensa attività di ricerca scientifica, supportata da crescenti evidenze, in particolare in modelli animali e sperimentali, di un coinvolgimento nella perturbazione degli assi endocrini e della fisiopatologia delle ghiandole endocrine, compresa la gonade maschile [4].

Il termine IE comprende un gruppo eterogeneo di sostanze naturali e di sintesi, che differiscono per sorgente di provenienza, caratteristiche ed effetti, tra cui plastiche e plastificanti [es. bisfenolo A (BPA), ftalati], solventi e prodotti industriali e loro sottoprodotti [es. diossine, bifenili policlorurati (PCB)], prodotti agricoli (es. pesticidi, insetticidi, fungicidi, erbicidi, fitoestrogeni), metalli pesanti [es. mercurio $(\mathrm{Hg})$, piombo $(\mathrm{Pb})$, cadmio $(\mathrm{Cd})$ ] [4]. Le vie di esposizione agli IE sono eterogenee e comprendono il contatto diretto e l'esposizione indiretta mediante disseminazione ambientale e/o transgenerazionale; pertanto, l'esposizione può comprendere una o più vie tra assorbimento cutaneo, 
Tabella 1 Classe di appartenenza, fonti e vie di esposizione relativi agli interferenti endocrini oggetto della rassegna

\begin{tabular}{|c|c|c|}
\hline $\begin{array}{l}\text { Interferente endocrino/classe di } \\
\text { appartenenza }\end{array}$ & Fonte & Via di esposizione \\
\hline Bisfenolo A / bisfenoli & $\begin{array}{l}\text { Plastiche in policarbonato; resine epossidiche; giocattoli } \\
\text { di plastica e bottiglie; lattine di alimenti }\end{array}$ & $\begin{array}{l}\text { Ingestione, inalazione, } \\
\text { assorbimento transdermico }\end{array}$ \\
\hline Ftalati / plastificanti & $\begin{array}{l}\text { Cibi contaminati; plastica e rivestimento per pavimenti in } \\
\text { polivinilcloruro; prodotti per la cura e igiene personale; } \\
\text { dispositivi medici; tubi }\end{array}$ & $\begin{array}{l}\text { Ingestione, inalazione, } \\
\text { assorbimento transdermico }\end{array}$ \\
\hline $\begin{array}{l}\text { 2,3,7,8-tetrachlorodibenzo-p-dioxin / } \\
\text { diossine }\end{array}$ & $\begin{array}{l}\text { Sottoprodotto della produzione di erbicidi clorurati; } \\
\text { fonderie; processo di sbiancamento della carta con cloro }\end{array}$ & Ingestione, inalazione \\
\hline $\begin{array}{l}\text { Bifenili policlorurati / } \\
\text { organoclorurati }\end{array}$ & $\begin{array}{l}\text { Aria e cibi contaminati; impianti e attrezzature elettriche } \\
\text { obsolete }\end{array}$ & $\begin{array}{l}\text { Ingestione, inalazione, } \\
\text { assorbimento transdermico }\end{array}$ \\
\hline Mercurio / metalli pesanti & $\begin{array}{l}\text { Cibi contaminati (pesce); interruttori, termostati, batterie, } \\
\text { amalgama dentale, soda caustica; reattori nucleari; } \\
\text { pesticidi e agenti antimicotici per la lavorazione del legno; } \\
\text { vernici }\end{array}$ & $\begin{array}{l}\text { Ingestione, inalazione, } \\
\text { assorbimento transdermico }\end{array}$ \\
\hline Piombo / Metalli pesanti & $\begin{array}{l}\text { Acqua e cibi contaminati; combustibili fossili; benzina; } \\
\text { vernici residenziali; batterie al piombo, munizioni; sistemi } \\
\text { idraulici, saldature e tubi; dispositivi per isolamento } \\
\text { elettrico e schermatura raggi X; vernici e pigmenti; lattine, } \\
\text { contenitori di ceramica e vetro; prodotti chimici }\end{array}$ & Ingestione, inalazione \\
\hline Cadmio / Metalli pesanti & $\begin{array}{l}\text { Acqua e cibi contaminati; fumo di sigaretta; batterie; } \\
\text { vernici e pigmenti; stabilizzanti plastici; pesticidi e } \\
\text { fertilizzanti; dispositivi fotovoltaici; lavorazione della } \\
\text { gomma e di altri metalli; zincatura; combustibili fossili; } \\
\text { discariche; incenerimento rifiuti }\end{array}$ & Ingestione, inalazione \\
\hline
\end{tabular}

inalazione, ingestione (cibo e acqua contaminati), passaggio transplacentare, allattamento al seno [4].

Alcune caratteristiche degli IE rendono particolarmente complessa l'interpretazione degli effetti e l'inferenza di una causalità specifica e di un rapporto dose-effetto, così come rendono al contempo preoccupante l'esposizione anche a bassi ma persistenti livelli di IE: 1) l'azione degli IE insiste su un substrato ormonale endogeno che può essere di per sé diversificato nella popolazione, esercitando, pertanto, effetti differenziali dose-dipendenti; 2) l'esposizione contemporanea a più IE e/o i fenomeni di bioaccumulo e biomagnificazione determinano l'instaurarsi di un'azione "cocktail", strettamente dipendente dal tipo e dalle dosi di IE compresenti, che possono esercitare tra loro effetti agonistici (sinergismo o additività) o antagonistici; 3) IE con debole attività ormonale possono indurre significative alterazioni per la compresenza di IE con simili caratteristiche, rendendo complessa la definizione di dosi soglia e/o curve dose-effetto; 4) gli IE possono esercitare effetti opposti a basse o alte dosi per il fenomeno di ormesi; 5) l'esistenza di finestre temporali di suscettibilità differenziale nel corso della vita, nonché l'eventuale latenza di effetti ed esordio di malattia, modulano l'entità delle alterazioni indotte e complicano l'interpretazione delle associazioni osservate; 6) l'impossibilità di effettuare studi di intervento mirati sull'uomo rende necessario il ricorso a studi preclinici in vitro e/o in modelli animali per la delucidazione dei meccanismi d'azione, con tutti i li- miti correlati alla traslazione animale-uomo delle evidenze sperimentali [4].

Nonostante le suddette criticità, i principali meccanismi d'azione che sottendono agli effetti endocrino-mediati degli IE sono stati ad oggi identificati, e includono il meccanismo d'azione principale, innescato dal legame degli IE ai recettori nucleari degli ormoni endogeni, inclusi il recettore degli androgeni (AR) e degli estrogeni (ER), di cui imitano o bloccano l'azione, e meccanismi mediati da recettori non steroidei, o non mediati da recettore, che includono interferenza con l'attività degli enzimi della steroidogenesi, con il metabolismo degli steroidi, con vie di segnalazione intracellulare, ciclo cellulare e apoptosi, ed effetti epigenetici nella linea somatica e germinale, questi ultimi coinvolti anche nella trasmissione transgenerazionale delle alterazioni indotte dagli IE [4]. Gli IE oggetto della presente rassegna con indicazione delle classi di appartenenza, fonti e vie di esposizione sono riportati in Tabella 1.

\section{Interferenti endocrini, steroidogenesi testicolare e fertilità maschile}

L'asse ipotalamo-ipofisi-testicolo e la funzione gonadica maschile, in entrambe le sue espressioni di steroidogenesi testicolare e spermatogenesi, sono tra i target maggiormente condizionati dalle azioni delle sostanze esogene ormono- 
mimetiche, in particolare degli IE con attività estrogenica $\mathrm{e}$ anti-androgenica [4]. L'esposizione prenatale a IE, durante una fase di elevata suscettibilità alle alterazioni ormonali in cui entrambe le funzioni del testicolo (steroidogenesi e spermatogenesi) vengono attivate, è stata proposta come fattore eziologico di alterazioni dello sviluppo e funzione della gonade maschile fetale, che determinano l'insorgenza di uno spettro di disturbi della funzione riproduttiva ricompresi nella sindrome da disgenesia testicolare: criptorchidismo, ipospadia, ipotestosteronemia, alterata spermatogenesi e infertilità, aumentato rischio di cancro testicolare [4]; pertanto, gli effetti degli IE sullo sviluppo del testicolo, in particolar modo durante la vita fetale, sono cruciali per la funzione riproduttiva in età adulta, la cui compromissione può essere permanente [5]. Tuttavia, benché i meccanismi d'azione degli IE sulla gonade maschile siano stati ampiamente descritti in modelli sperimentali, gli effetti dell'esposizione umana agli IE in età adulta sono ancora controversi, a causa di una scarsa letteratura dedicata e delle predette limitazioni.

\section{Bisfenolo A}

Diversi studi hanno valutato l'effetto dell'esposizione al BPA su qualità del liquido seminale e funzione endocrina testicolare; tuttavia, nonostante chiare evidenze sperimentali, i dati clinici sono controversi [6]. In particolare, in soggetti con esposizione occupazionale è stata evidenziata una correlazione negativa tra i livelli urinari di BPA e concentrazione, conta totale, vitalità e motilità spermatica, sebbene in soggetti con esposizione ambientale tale correlazione fosse mantenuta solo per concentrazione e conta totale [7]; inoltre, uno studio di coorte su soggetti infertili ha riportato un'associazione tra elevati livelli urinari di BPA e ridotta concentrazione, motilità e morfologia tipica degli spermatozoi, e una correlazione positiva con il danno al DNA degli spermatozoi [8]. Tuttavia, altri studi su partner maschili di coppie infertili non hanno riportato associazioni significative tra i livelli urinari di BPA e ridotta qualità seminale, e i livelli di BPA non sembrano correlare con gli outcome riproduttivi in coppie arruolate in percorsi di PMA [6].

Ancor più eterogenea è l'associazione dell'esposizione al BPA con il profilo degli steroidi sessuali. Infatti, ridotti livelli di ormone follicolo stimolante (FSH) ma non ormone luteinizzante (LH) e testosterone libero sono stati riportati in soggetti con esposizione occupazionale al BPA, mentre nella popolazione generale è stata riportata una correlazione positiva con i livelli di testosterone ma non $17-\beta$ estradiolo, SHBG o testosterone libero, sebbene un diverso studio abbia evidenziato un livello significativamente più elevato di testosterone totale e libero, LH e 17- $\beta$ estradiolo nel quartile superiore di BPA urinario [6]. Studi su coorti di soggetti infertili hanno riportato dati parzialmente discordanti. In particolare, uno studio ha evidenziato che i livelli urinari di
BPA erano positivamente correlati ai livelli di SHBG e negativamente correlati con l'indice di androgeni liberi (FAI) e il rapporto FAI/LH, sebbene non correlati con FSH, LH, testosterone totale e libero e inibina B [9]. Un secondo studio ha invece riportato una correlazione negativa tra i livelli urinari di BPA e inibina $\mathrm{B}$ e rapporto $17-\beta$ estradiolo/testosterone e una correlazione positiva con FSH e rapporto FSH/inibina $\mathrm{B}[8]$.

Nonostante l'incoerenza degli studi clinici, studi in modelli animali e in vitro hanno individuato potenziali meccanismi d'azione alla base della potenziale disfunzione gonadica maschile; il trattamento con BPA impatta su spermatogenesi e steroidogenesi testicolare maggiormente per interferenza endocrina con l'asse ipotalamo-ipofisi-testicolo e per inibizione diretta dell'attività della proteina StAR e degli enzimi steroidogenici 3 - $\beta$-idrossisteroide deidrogenasi e $17 \beta$-idrossisteroide deidrogenasi, induzione dell'iperespressione dell'aromatasi, riduzione del numero di cellule germinali, del Sertoli, e di Leydig indotta da iperespressione dei mediatori apoptotici Fas e Fas ligando a livello testicolare, alterazione del signaling di glucosio e insulina e dell'attività mitocondriale in cellule germinali e di Leydig, e disfunzione del sistema antiossidante a livello testicolare con conseguente stress ossidativo (Fig. 1) [6, 10].

\section{Ftalati}

Diversi studi hanno valutato l'effetto dell'esposizione agli ftalati su qualità del liquido seminale e funzione endocrina testicolare, riportando un grado differenziale di associazione con la disfunzione gonadica maschile [11]. Le evidenze più coerenti e robuste riportate da una meta-analisi recente su studi di popolazione o di coorte, non limitati a soggetti con problemi di fertilità, sono a favore di un'associazione tra esposizione agli ftalati DBP, BBP, DEHP e DINP e ridotta concentrazione di spermatozoi. L'esposizione a DIBP e DEP non è stata invece associata alla riduzione della qualità dei parametri seminali, nel primo caso probabilmente per mancanza di sufficienti studi [11]. Lo stesso studio di meta-analisi ha riportato una moderata evidenza di associazione tra esposizione agli ftalati DEHP, DINP e DIBP e ridotti livelli di testosterone, e una debole evidenza per DBP. L'esposizione al BBP e DEP non è associata ad alterazioni dei livelli di testosterone, nel primo caso probabilmente a causa dei bassi livelli di esposizione e scarsa sensibilità di dosaggio negli studi dedicati [11].

Studi sperimentali in modelli animali dimostrano che gli ftalati, agendo comunemente in cocktail compositi, impattano sulla funzione della gonade maschile mediante azioni locali e centrali; in particolare, il trattamento con DEHP, somministrato come singolo composto ad alte dosi oppure a basse dosi in combinazione con altri ftalati (DMP, DEP, DBP, BBP e DNOP) e il DBP, inducono una riduzione significativa dei livelli di testosterone e $\mathrm{LH}$ e degli enzimi 
Fig. 1 Meccanismi d'azione del bisfenolo A (BPA) sulla steroidogenesi testicolare: interferenza con l'azione della proteina StAR e degli enzimi steroidogenici

3- $\beta$-idrossisteroide deidrogenasi (3 $\beta$-HSD) e $17 \beta$-idrossisteroide deidrogenasi (17 $\beta$-HSD)

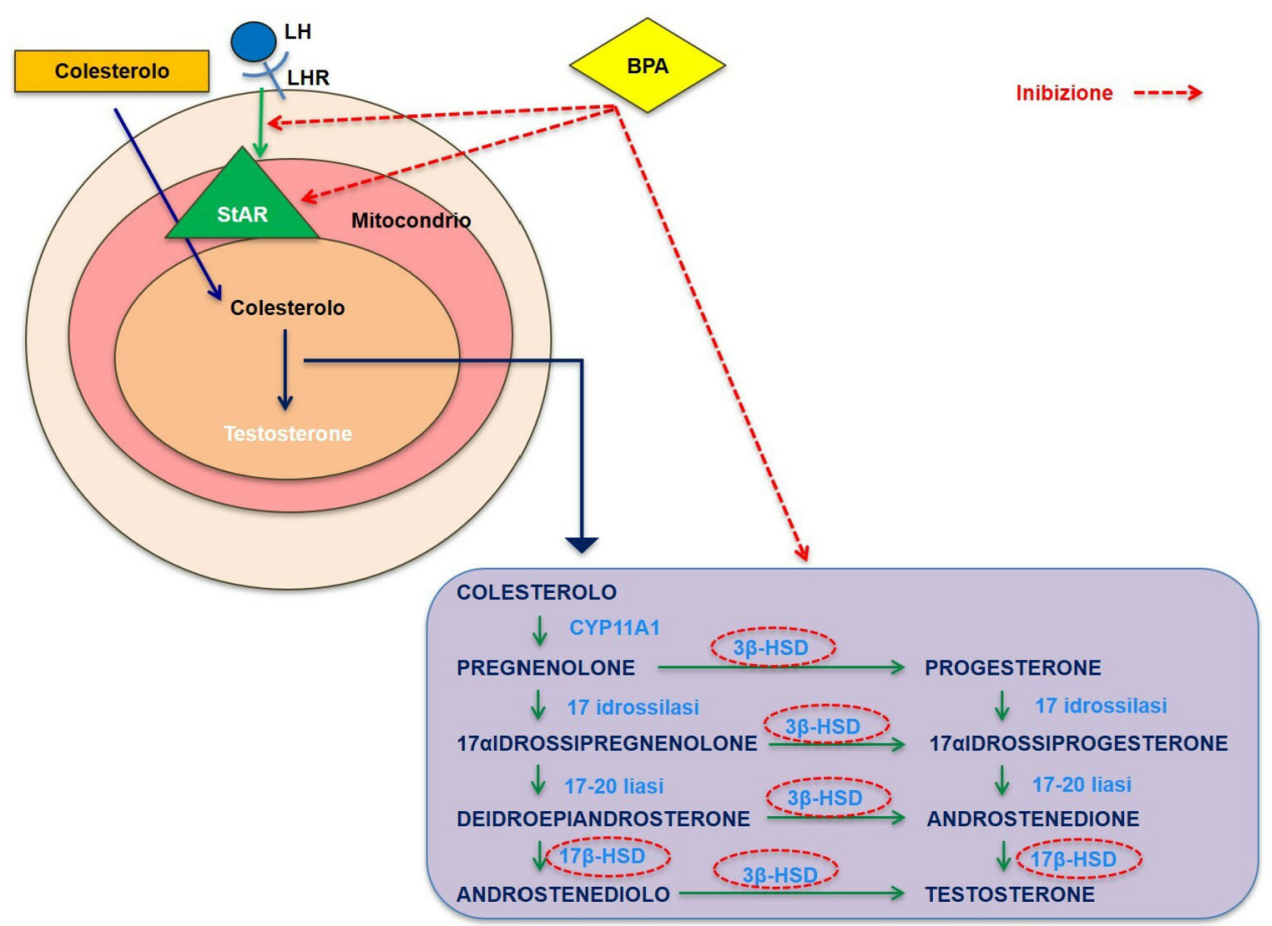

della steroidogenesi testicolare, alterazioni morfostrutturali dei tubuli seminiferi fino ad assenza di cellule germinali, rottura delle giunzioni aderenti tra le cellule di Sertoli, e un incremento significativo dello stress ossidativo, suggerendo la sussistenza di effetti sinergici tra tipologie chimicamente differenti di ftalati, sui medesimi targets molecolari [12-14].

\section{Diossine}

Pochi studi hanno valutato l'effetto dell'esposizione alle diossine su qualità del liquido seminale e funzione endocrina testicolare, suggerendo un' associazione con un profilo seminale ed endocrino patologico e infertilità. Uno studio condotto su giovani maschi con esposizione perinatale tramite latte materno di donne esposte a diossina in occasione del disastro industriale di Seveso (Italia), ha evidenziato una significativa riduzione di concentrazione, conta totale e motilità totale e progressiva degli spermatozoi, rispetto a giovani maschi le cui madri non erano state esposte [15]. Tali evidenze suggeriscono un effetto permanente delle diossine sulla qualità seminale, connesso a un preciso timing di esposizione coincidente con la fase di espansione del numero di cellule del Sertoli; tale ipotesi è corroborata dalla mancanza di associazione tra esposizione in età adulta e ridotta qualità seminale, nella popolazione generale [15]. Tuttavia, un interessante studio in soggetti residenti in un'area a elevata contaminazione da diossine ha dimostrato livelli significativamente superiori di diossine nell'eiaculato di uomini infertili, rispetto a donatori fertili, e livelli significativamente superiori di 2,3,7,8-tetrachlorodibenzo-p-dioxin (TCDD), la più tossica tra le diossine, nell'eiaculato di uomini infertili con alterazioni seminali rispetto agli infertili normozoospermici [16].

Uno studio recente condotto su coppie madre-figlio ha dimostrato livelli significativamente ridotti di testosterone e deidroepiandrosterone (DHEA) nel siero e una ridotta attività degli enzimi steroidogenici $17 \alpha$-idrossilasi e $17 \beta$ idrossisteroide deidrogenasi in bambini nati da madri residenti in un'area a elevata contaminazione da diossine, rispetto a bambini nati in zone controllo, e una correlazione negativa dei due ormoni con i livelli di diossine nel latte materno [17]. Coerentemente, in uomini residenti in aree a elevata contaminazione da diossine è stata riportata una correlazione negativa tra i livelli di diossine e testosterone, ma non gonadotropine e progesterone [18].

Modelli in vitro ed in vivo hanno dimostrato che i composti diossina-simili e il TCDD legano il recettore degli idrocarburi arilici (AhR), un fattore di trascrizione citoplasmatico che, in seguito a legame con il ligando e traslocazione nel nucleo, forma un complesso con l'AhR nuclear translocator (ARNT) e regola l'espressione di geni bersaglio, compresi geni estrogeno-dipendenti, esercitando, pertanto, azioni estrogeno-mimetiche che possono interferire con il sistema endocrino endogeno [19]. Inoltre, in modelli animali è stato dimostrato che il trattamento con TCDD riduce significativamente conta totale, motilità e morfologia tipica degli spermatozoi, nonché l'espressione negli spermatozoi della proteina CatSper, implicata nella regolazione della motilità e della penetrazione della zona pellucida, ed è stata documentata degenerazione necrotica del testicolo con ridotto 
spessore dell'epitelio seminifero, correlati all'aumento dei radicali liberi dell'ossigeno e allo stress ossidativo, mediati dalla riduzione dell'espressione di superossido dismutasi e catalasi [20].

\section{Policlorobifenili}

Diversi studi hanno valutato l'effetto dell'esposizione ai PCB su qualità del liquido seminale e funzione endocrina testicolare, ma le evidenze cliniche sono eterogenee, in particolare in relazione ai parametri seminali. La maggioranza degli studi condotti su popolazione generale e partners maschili di coppie infertili ha mostrato una correlazione negativa tra esposizione ai PCB e motilità spermatica, mentre evidenze più scarse e controverse sono riportate relativamente alla morfologia [4]; inoltre, in uno studio caso-controllo in pazienti con cancro testicolare, la prevalenza di oligozoospermia era più elevata nei pazienti con livelli dosabili di PCB, rispetto ai pazienti con livelli indosabili [21]. Infine, alcuni studi trasversali hanno dimostrato un' associazione positiva tra l'esposizione ai PCB (CB-153) e l'indice di frammentazione del DNA spermatico, e l'espressione di fattori anti-apoptotici (quale meccanismo compensatorio), nella popolazione europea ma non nella popolazione fortemente esposta degli Inuit, suggerendo un ruolo dell'esposizione ai PCB nella compromissione dell'integrità cromatinica degli spermatozoi, sebbene non si possano escludere concause genetiche e legate allo stile di vita [22, 23].

Più scarsa la letteratura scientifica dedicata ai possibili effetti dell'esposizione ai PCB e la funzione endocrina testicolare; uno studio ha mostrato livelli sierici di testosterone ridotti in ragazzi e adolescenti esposti ai PCB, rispetto ai non esposti [24], e l'esposizione ai PCB era negativamente correlata ai livelli di testosterone totale, libero, diidrotestosterone e al FAI in uomini con diverso grado di fertilità [25]. Uno studio ampio su una coorte di giovani pescatori in un'area a forte esposizione ai $\mathrm{PCB}$ ha riportato una correlazione positiva tra i livelli sierici di PCB e di SHBG, LH, testosterone totale e rapporto testosterone/17- $\beta$ estradiolo, con un meccanismo ipotizzato di danno diretto alle cellule di Leydig e conseguente aumento di LH, e un incremento compensatorio di testosterone in risposta ai livelli elevati di SHBG [26]. Queste evidenze sono apparentemente confermate da uno studio su giovani valutati durante visita di leva per il servizio militare, che mostrava una correlazione positiva dei livelli di PCB con SHBG e una correlazione negativa con il rapporto testosterone/SHBG [27].

Modelli in vitro e in vivo hanno dimostrato effetti diretti sulla vitalità e steroidogenesi nelle cellule di Leydig: i PCB legano il AhR e interferiscono con espressione e attività degli enzimi steroidogenici, in particolare StAR, citocromo P450scc, $3 \beta$-idrossisteroide deidrogenasi e $17 \beta$ idrossisteroide deidrogenasi, e riducono l'espressione dei recettori per LH. Infine, è stata dimostrata una significativa induzione della produzione di radicali liberi dell'ossigeno conseguente a inibizione dell'attività degli enzimi antiossidanti, tra cui superossido-dismutasi, glutatione-reduttasi, glutatione-perossidasi e catalasi [28]. Meccanismi analoghi sono stati dimostrati in spermatozoi umani e animali: in particolare, il trattamento in vivo e/o in vitro con un mix di PCB riduce la proliferazione e induce apoptosi nelle cellule germinali, eventi accompagnati da un aumento significativo delle specie reattive dell'ossigeno, da incrementata frammentazione del DNA spermatico e da ridotte conta totale e motilità degli spermatozoi [29-31].

\section{Metalli pesanti}

Numerosi studi in vitro e in modelli animali hanno fornito consistenti evidenze di un effetto deleterio dei metalli pesanti su spermatogenesi e steroidogenesi, sebbene le evidenze cliniche in merito al ruolo dell'esposizione ambientale e occupazionale siano più scarse.

\section{Mercurio}

Il ruolo dell'esposizione al $\mathrm{Hg}$ sulla funzione gonadica maschile è tuttora poco chiaro negli studi clinici; studi condotti in coorti di pazienti subfertili o infertili hanno mostrato una correlazione negativa tra i livelli di $\mathrm{Hg}$ e la concentrazione, conta totale, motilità e morfologia tipica, sebbene altri studi in coorti piccole e con bassa esposizione non abbiano confermato tali risultati, probabilmente a causa di limitazioni tecniche legate al disegno di studio; va tuttavia considerato che gli studi con forte evidenza di associazione con una peggiore qualità seminale sono stati principalmente condotti in popolazioni con un consumo ingente di prodotti ittici, caratterizzate dunque da un elevato rischio di cross-contaminazione con altri inquinanti [32].

Studi in modelli sperimentali hanno mostrato che l'esposizione al $\mathrm{Hg}$ induce danni strutturali e funzionali diretti nelle cellule di Leydig con inibizione dell'attività degli enzimi della steroidogenesi ( $3 \beta$-idrossisteroide deidrogenasi), riducendo la produzione di testosterone; effetti diretti sulle cellule del Sertoli determinano una riduzione della produzione di inibina B. Studi in vitro hanno dimostrato che gli effetti sulla motilità spermatica sono plausibilmente indotti da interferenza con il sistema di scivolamento dineina/microtubuli e dall'incremento della perossidazione lipidica della membrana degli spermatozoi e frammentazione del DNA spermatico; inoltre, è stata dimostrata anche una ridotta vitalità e capacità di reazione acrosomiale [32].

\section{Piombo}

Numerosi studi clinici in uomini con esposizione occupazionale o ambientale $\mathrm{al} \mathrm{Pb}$ hanno dimostrato in modo piuttosto 
coerente che l'esposizione $\mathrm{al} \mathrm{Pb}$ è associata a un peggioramento della qualità del liquido seminale; in particolare, in soggetti infertili sono stati dimostrati livelli significativamente più elevati di $\mathrm{Pb}$, rispetto a soggetti fertili, ed è stata ripetutamente riportata una correlazione negativa tra i livelli di $\mathrm{Pb}$ ematico e/o seminale e la concentrazione, conta totale, motilità e morfologia tipica [32-34]. Inoltre, in pazienti arruolati in PMA, i livelli seminali di $\mathrm{Pb}$ erano correlati negativamente con il rate di fecondazione [32]. Scarsi sono gli studi che hanno valutato l'effetto dell'esposizione al $\mathrm{Pb}$ sul profilo degli steroidi sessuali, e sembrano suggerire che anche in un setting di elevata esposizione al $\mathrm{Pb}$ (occupazionale) non vi siano associazioni con i livelli di testosterone o gonadotropine [32, 35].

Studi in modelli animali hanno mostrato che il $\mathrm{Pb}$ può indurre effetti diretti sul testicolo, determinando atrofia testicolare e alterazioni di spermatogenesi e steroidogenesi, ed effetti indiretti sull'asse ipotalamo-ipofisi-testicolo; una specifica tossicità è stata dimostrata verso le cellule di Leydig, ma non del Sertoli. I meccanismi che sottendono agli effetti tossici del $\mathrm{Pb}$ includono la sostituzione calcio- $\mathrm{Pb}$ nelle giunzioni aderenti della barriera emato-testicolare; il displacement dello zinco nelle metallotioneine con conseguente riduzione della biodisponibilità di zinco; induzione di stress ossidativo, perossidazione lipidica, frammentazione del DNA e apoptosi e inibizione dei sistemi di riparazione del DNA, danno all'endotelio vascolare [32].

\section{Cadmio}

Numerosi studi clinici suggeriscono che l'esposizione anche a bassi livelli (ambientali) di Cd possa essere associata a ridotta qualità del liquido seminale [36]; in particolare, livelli di $\mathrm{Cd}$ più elevati sono stati misurati nei fluidi biologici di soggetti infertili o con parametri seminali patologici, e i livelli di $\mathrm{Cd}$ in diverse matrici biologiche correlano negativamente con tutti i parametri seminali convenzionali e positivamente con i livelli di 8-idrossi-2-deossiguanosina, un marcatore di stress ossidativo, sebbene vi sia un certo grado di eterogeneità in funzione della matrice biologica considerata [36]. Questa eterogeneità appare parzialmente spiegata da una tendenza all'accumulo di $\mathrm{Cd}$ nel liquido seminale, che renderebbe pertanto questa matrice biologica più idonea per la valutazione oggettiva degli effetti del Cd sui parametri seminali [36].

Decisamente più scarsi sono gli studi relativi alla funzione endocrina testicolare, che riportano eterogeneamente una mancanza di associazione o una associazione positiva tra i livelli di $\mathrm{Cd}$ in diverse matrici biologiche e i livelli di testosterone, e una ancor maggiore eterogeneità nel tipo di associazione tra livelli di $\mathrm{Cd}$ e gonadotropine [36]. Nonostante l'eterogeneità degli studi clinici, complessivamente a favore di un effetto negativo dell'esposizione al $\mathrm{Cd}$ sulla funzione testicolare, i meccanismi di danno Cd-indotto a carico di spermatogenesi e steroidogenesi, inequivocabilmente dimostrati principalmente in modelli animali e in $v i$ tro, sono molteplici e includono: danno diretto strutturale e funzionale del testicolo mediato da disfunzione dell'endotelio vascolare dell'arteria spermatica interna, dei rami epididimali e del plesso pampiniforme, che determina edema, emorragia, infiammazione, ipossia e necrosi testicolare; destabilizzazione e compromissione dell'integrità della barriera emato-testicolare; induzione di infiammazione e apoptosi; citotossicità Sertoli- e Leydig-diretta; interferenza e mimetismo molecolare con zinco e selenio, elementi essenziali per la funzione riproduttiva maschile e parte integrante dei sistemi antiossidanti, in particolare del sistema del glutatione e della glutatione perossidasi, che determina un incremento indiretto Cd-indotto dello stress ossidativo e danno al DNA nelle cellule di Leydig e nella linea germinale; riduzione dell'espressione dei recettori del LH e degli enzimi della steroidogenesi StAR, citocromo P450scc e $17 \beta$-idrossisteroide deidrogenasi (Fig. 2) [36].

\section{Conclusioni}

La progressiva riduzione della fertilità di coppia e della qualità del liquido seminale negli ultimi decenni, l'eterogeneità geografica della qualità del liquido seminale e le evidenze sperimentali di un'azione deleteria sugli assi endocrini e sul la fisiologia della gonade maschile, hanno determinato un crescente interesse nel potenziale effetto dell'esposizione agli inquinanti ambientali e, in particolare, agli IE su spermatogenesi e steroidogenesi. I principali meccanismi d'azione degli IE sono stati identificati principalmente in modelli sperimentali animali e in vitro e i dati epidemiologici e osservazionali sono limitati e spesso incoerenti a causa dell'eterogeneità delle popolazioni studiate e delle metodologie.

L'esposizione occupazionale al BPA è stata associata a una riduzione della qualità del liquido seminale, confermata solo in parte da studi su soggetti con esposizione ambientale, mentre studi di coorte su soggetti infertili riportano dati controversi. L'esposizione a diverse classi di ftalati mostra effetti deleteri principalmente in relazione alla concentrazione degli spermatozoi, con più limitate evidenze per altri parametri seminali, e una moderata evidenza di associazione tra esposizione ad alcune classi di ftalati e ridotti livelli di testosterone. I pochi studi disponibili in merito alle diossine evidenziano un potenziale effetto deleterio permanente dell'esposizione in età perinatale sulla qualità seminale nella popolazione generale, suggerendo l'importanza del timing nella suscettibilità agli effetti riproduttivi della diossina; tuttavia, in popolazioni con elevati livelli di esposizione ambientale alle diossine, ma non nella popolazione generale, l'esposizione in età adulta è stata associata a una peggiore qualità seminale. 
Fig. 2 Panoramica dei meccanismi patogenetici proposti per la tossicità riproduttiva del cadmio. In carattere rosso sono riportati i principali meccanismi patogenetici, con indicazione del corrispondente organo/cellula bersaglio. Il termine "testicolo" si riferisce a meccanismi patogenetici dimostrati da esperimenti in vivo, o su omogenati di tessuto testicolare intero. I rettangoli in azzurro rappresentano l'effetto finale proposto/ipotizzato sulla funzione riproduttiva maschile. Tradotto e adattato da [36]

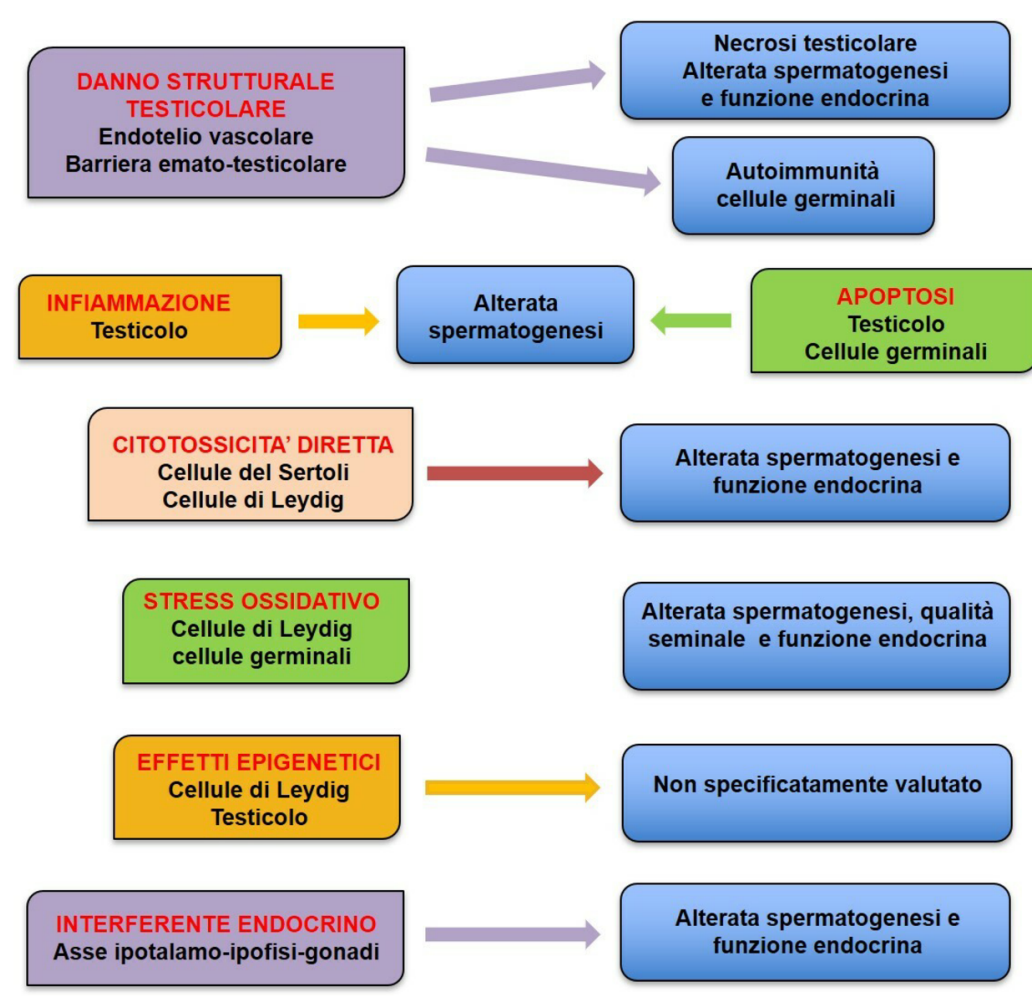

L'esposizione a elevati livelli di diossina si associa a ridotti livelli di testosterone, indipendentemente dal timing di esposizione. La maggioranza degli studi nella popolazione generale e in uomini infertili mostra una correlazione negativa tra livello di esposizione ai PCB e motilità spermatica, e un' associazione con elevato indice di frammentazione del DNA spermatico; alcune evidenze in soggetti della popolazione generale con esposizione ai PCB mostrano livelli sierici di testosterone ridotti, mentre in soggetti con esposizione elevata una correlazione positiva tra i livelli sierici di PCB e i livelli di SHBG, LH e testosterone totale rende ipotizzabile un meccanismo di danno diretto alle cellule di Leydig e conseguente aumento compensatorio di LH e testosterone in risposta ai livelli elevati di SHBG.

Studi principalmente condotti in popolazioni con un elevato consumo di prodotti ittici mostrano che livelli elevati di $\mathrm{Hg}$ sono associati a ridotta qualità seminale, sebbene una cross-contaminazione con altri inquinanti dei prodotti alimentari consumati non possa essere esclusa, determinando una certa incertezza nelle evidenze. L'esposizione occupazionale o ambientale al $\mathrm{Pb}$ e al $\mathrm{Cd}$ è associata in modo piuttosto coerente a un peggioramento della qualità del liquido seminale e, per il $\mathrm{Pb}$, un ridotto rate di fecondazione in PMA; di contro, livelli anche occupazionali di esposizione al $\mathrm{Pb}$ non sembrano essere associati ad alterazioni dei livelli di testosterone o gonadotropine, mentre una marcata eterogeneità caratterizza il rapporto tra esposizione al Cd e livelli di questi ormoni.
Sono necessari ulteriori studi longitudinali e multicentrici in diverse aree geografiche e popolazioni esposte, esaustivamente controllati per fattori di confusione ed esposizioni multiple. Inoltre, gli effetti transgenerazionali padre-figlio sono meritevoli di particolare attenzione, al fine di determinare il potenziale ruolo degli IE sulla funzione riproduttiva maschile nei discendenti della popolazione esposta.

Informazioni Supplementari La versione online contiene materiale supplementare disponibile su https://doi.org/10.1007/s40619-02201012-9.

Ringraziamenti Gli autori ringraziano Davide Menafra e Francesco Garifalos per il contributo nella scrittura della rassegna, Claudia Veneruso e Maria Maddalena Passarelli per il contributo nella preparazione delle figure.

\section{Dichiarazioni etiche}

Conflitto di interesse Gli autori Cristina de Angelis, Marco Mazzella e Rosario Pivonello dichiarano di non avere conflitti di interesse.

Consenso informato Lo studio presentato in questo articolo non ha richiesto sperimentazione umana.

Studi sugli animali Gli autori di questo articolo non hanno eseguito studi sugli animali.

Open Access This article is licensed under a Creative Commons Attribution 4.0 International License, which permits use, sharing, adaptation, distribution and reproduction in any medium or format, as long as you give appropriate credit to the original author(s) and the source, 
provide a link to the Creative Commons licence, and indicate if changes were made. The images or other third party material in this article are included in the article's Creative Commons licence, unless indicated otherwise in a credit line to the material. If material is not included in the article's Creative Commons licence and your intended use is not permitted by statutory regulation or exceeds the permitted use, you will need to obtain permission directly from the copyright holder. To view a copy of this licence, visit http://creativecommons.org/licenses/by/4.0/.

\section{Bibliografia}

1. WHO (2020) Sexual and reproductive health. https://www.who. int/reproductivehealth/topics/infertility/multiple-definitions/en/

2. Carlsen E, Giwercman A, Keiding N, Skakkebaek NE (1992) Evidence for decreasing quality of semen during past 50 years. BMJ 305(6854):609-613

3. Nordkap L, Nordström Joensen U, Blomberg Jensen M, Jørgensen N (2012) Regional differences and temporal trends in male reproductive health disorders: semen quality may be a sensitive marker of environmental exposures. Mol Cell Endocrinol 355(2):221-230

4. Gore AC, Chappell VA, Fenton SE et al (2015) EDC-2: The Endocrine Society's second scientific statement on endocrinedisrupting chemicals. Endocr Rev 36(6):E1-E150

5. Rouiller-Fabre V, Habert R, Livera G (2014) Effects of endocrine disruptors on the human fetal testis. Ann Endocrinol (Paris) 75(2):54-57

6. De Toni L, De Rocco Ponce M, Cosmin Petre G et al (2020) Bisphenols and male reproductive health: from toxicological models to therapeutic hypotheses. Front Endocrinol (Lausanne) 11:301

7. Li DK, Zhou ZJ, Miao M et al (2011) Urine bisphenol-A (BPA) level in relation to semen quality. Fertil Steril 95(2):625-630

8. Meeker JD, Barr DB, Hauser R (2008) Human semen quality and sperm DNA damage in relation to urinary metabolites of pyrethroid insecticides. Hum Reprod 23(8):1932-1940

9. Mendiola J, Jørgensen N, Andersson A-M et al (2010) Are environmental levels of bisphenol a associated with reproductive function in fertile men? Environ Health Perspect 118(9):1286-1291

10. Barbagallo F, Condorelli RA, Mongioì LM et al (2020) Effects of bisphenols on testicular steroidogenesis. Front Endocrinol (Lausanne) 11:373

11. Radke EG, Braun JM, Meeker JD, Cooper GS (2018) Phthalate exposure and male reproductive outcomes: a systematic review of the human epidemiological evidence. Environ Int 121(Pt(1):764-793

12. Wang Y, Ni C, Li X et al (2019) Phthalate-induced fetal Leydig cell dysfunction mediates male reproductive tract anomalies. Front Pharmacol 10:1309

13. Latini G, Del Vecchio A, Massaro M et al (2006) Phthalate exposure and male infertility. Toxicology 226(2-3):90-98

14. Pallotti F, Pelloni M, Gianfrilli D et al (2020) Mechanisms of testicular disruption from exposure to bisphenol A and phtalates. $\mathrm{J}$ Clin Med 9(2):471

15. Mocarelli P, Gerthoux PM, Needham LL et al (2011) Perinatal exposure to low doses of dioxin can permanently impair human semen quality. Environ Health Perspect 119(5):713-718

16. Galimova EF, Amirova ZK, Galimov N (2015) Dioxins in the semen of men with infertility. Environ Sci Pollut Res Int 22(19):14566-14569

17. Oanh NT, Kido T, Honma S et al (2018) Androgen disruption by dioxin exposure in 5-year-old Vietnamese children: decrease in serum testosterone level. Sci Total Environ 640-641:466-474
18. Van Luong H, Tai PT, Nishijo M et al (2018) Association of dioxin exposure and reproductive hormone levels in men living near the Bien Hoa airbase. Vietnam Sci Total Environ 628-629:484-489

19. Tavakoly Sany SB, Hashim R, Salleh A et al (2015) Dioxin risk assessment: mechanisms of action and possible toxicity in human health. Environ Sci Pollut Res Int 22(24):19434-19450

20. Mohammadi S, Rahmani F, Hasanian SM et al (2019) Effects of dioxin on testicular histopathology, sperm parameters, and CatSper2 gene and protein expression in Naval Medical Research Institute male mice. Andrologia 51(11):e13411

21. Paoli D, Giannandrea F, Gallo M et al (2015) Exposure to polychlorinated biphenyls and hexachlorobenzene, semen quality and testicular cancer risk. J Endocrinol Invest 38(7):745-752

22. Spano $\mathrm{M}$ et al (2005) Exposure to PCB and p, p'-DDE in European and Inuit populations: impact on human sperm chromatin integrity. Hum Reprod 20(12):3488-3499

23. Stronati AM, Cecati M, Bordicchia M et al (2006) Relationships between sperm DNA fragmentation, sperm apoptotic markers and serum levels of CB-153 and p,p0 -DDE in European and Inuit populations. Reproduction 132:949-958

24. Schell LM, Gallo MV, Deane GD et al (2014) Relationships of polychlorinated biphenyls and dichlorodiphenyldichloroethylene (p,p'-DDE) with testosterone levels in adolescent males. Environ Health Perspect 122(3):304-309

25. Vitku J, Heracek J, Sosvorova L et al (2016) Associations of bisphenol A and polychlorinated biphenyls with spermatogenesis and steroidogenesis in two biological fluids from men attending an infertility clinic. Environ Int 89-90:166-173

26. Petersen MS, Halling J, Jørgensen N et al (2018) Reproductive function in a population of young Faroese men with elevated exposure to polychlorinated biphenyls (PCBs) and perfluorinated alkylate substances (PFAS). Int J Environ Res Public Health 15(9): 1880

27. Richthoff $J$ et al (2003) Serum levels of 2, 2',4, 4',5, 5'hexachlorobiphenyl (CB-153) in relation to markers of reproductive function in young males from the general Swedish population. Environ Health Perspect 111(4):409-413

28. Murugesan P, Balaganesh M, Balasubramanian K, Arunakaran J (2007) Effects of polychlorinated biphenyl (Aroclor 1254) on steroidogenesis and antioxidant system in cultured adult rat Leydig cells. J Endocrinol 192(2):325-338

29. Sumner RN, Tomlinson M, Craigon J et al (2019) Independent and combined effects of diethylhexyl phthalate and polychlorinated biphenyl 153 on sperm quality in the human and dog. Sci Rep 9(1):3409

30. Cai J, Wang C, Wu T et al (2011) Disruption of spermatogenesis and differential regulation of testicular estrogen receptor expression in mice after polychlorinated biphenyl exposure. Toxicology 287(1-3):21-28

31. Jiang LG, Cheng L-Y, Kong S-H et al (2017) Toxic effects of polychlorinated biphenyls (Aroclor 1254) on human sperm motility. Asian J Androl 19(5):561-566

32. Wirth JJ, Mijal RS (2010) Adverse effects of low level heavy metal exposure on male reproductive function. Syst Biol Reprod Med 56(2):147-167

33. Mendiola J, Moreno JM, Roca M et al (2011) Relationships between heavy metal concentrations in three different body fluids and male reproductive parameters: a pilot study. Environ Health 10(1):6

34. Pant N, Kumar G, Upadhyay AD et al (2015) Correlation between lead and cadmium concentration and semen quality. Andrologia 47(8):887-891

35. Balachandar R, Bagepally BS, Kalahasthi R, Haridoss M (2020) Blood lead levels and male reproductive hormones: a systematic review and meta-analysis. Toxicology 443:152574 
36. de Angelis C, Galdiero M, Pivonello C et al (2017) The environment and male reproduction: the effect of cadmium exposure on reproductive function and its implication in fertility. Reprod Toxicol 73:105-127
Nota della casa editrice Springer Nature rimane neutrale in riguardo alle rivendicazioni giurisdizionali nelle mappe pubblicate e nelle affiliazioni istituzionali. 